\title{
Tilted Beam Piezoresistive Displacement Sensor: Design, Modeling, and Characterization
}

\author{
Mohammad Maroufi, Student Member, IEEE, Ali Bazaei, Member, IEEE, Ali Mohammadi, Member, IEEE, \\ and S. O. Reza Moheimani, Fellow, IEEE
}

\begin{abstract}
We present a comprehensive study of the design, modeling, and characterization of an on-chip piezoresistive displacement sensor. The design is based on the bulk piezoresistivity of tilted clamped-guided beams without the need for additional steps to generate doped regions. The sensor is implemented in a one-degree-of-freedom microelectromechanical system (MEMS) nanopositioner, where the beams also function as the suspension system. A standard MEMS fabrication process is used to realize the device on single-crystalline silicon as the structural material. The beams are oppositely tilted to develop tensile and compressive axial forces during stage movement, creating a differential sensing feature. An analytical approach is proposed for modeling and design of the tilted clamped-guided beams. The linearity of the sensor in the differential configuration is investigated analytically. The static, dynamic, and noise characteristics of the sensor are presented, followed by a model-based investigation of the measured dynamic feedthrough.

[2015-0030]
\end{abstract}

Index Terms-Piezoresistive displacement sensor, MEMS nanopositioner, tilted clamped-guided beam, buckling, negative stiffness.

\section{INTRODUCTION}

$\mathbf{R}$ ECENT research on emerging micro-electromechanical systems (MEMS) devices such as nanopositioners and microgrippers necessitates the integration of displacement sensing into these systems [1]-[5]. These displacement sensors should have a small footprint and satisfy the noise, dynamic range and bandwidth requirements of the device [6], [7]. In addition, the required fabrication steps for the sensor should be compatible with the existing standard microfabrication processes to reduce the production cost. Sensing concepts such as capacitive [8], electrothermal [9] and piezoresistive [10] have been successfully implemented in a variety of MEMS devices.

Capacitive sensing is widely used to detect displacement of MEMS devices with a high bandwidth and resolution. Implementation of this technique is often compatible with

Manuscript received January 27, 2015; revised March 25, 2015; accepted April 14, 2015. Date of publication May 17, 2015; date of current version September 29, 2015. Subject Editor A. Zhang.

M. Maroufi and A. Bazaei are with the School of Electrical Engineering and Computer Science, University of Newcastle Australia, Callaghan, NSW 2308, Australia (e-mail: mohammad.maroufi@uon.edu.au; ali.bazaei@newcastle.edu.au).

A. Mohammadi is with the Department of Electrical and Computer Systems Engineering, Monash University, Clayton, VIC 3800, Australia (e-mail: mohamadi@ieee.org).

S. O. Reza Moheimani is with the Department of Mechanical Engineering, University of Texas at Dallas, Richardson, TX 75080 USA (e-mail: reza.moheimani@utdallas.edu).

Color versions of one or more of the figures in this paper are available online at http://ieeexplore.ieee.org.

Digital Object Identifier 10.1109/JMEMS.2015.2426180 standard microfabrication processes. However, capacitive sensors occupy a large fraction of the chip and may need complicated readout circuits to detect small capacitance variations [11], [12].

Electrothermal sensing is an alternative technology for on-chip position measurement in MEMS, where the displacement of a heat sink induces opposite resistance changes in a pair of heaters [13]-[15]. In comparison with the capacitive sensors, electrothermal sensors occupy a small foot print, and require a relatively simple readout circuit [16]. However, they have a limited bandwidth, and cannot be used in vacuum conditions.

Piezoresistivity of silicon has also been exploited in a variety of displacement sensing applications [17]. MEMS nanopositioners [18] and inertial sensors [8] are two examples of such applications.

Piezoresistive sensing is based on the variation of the electrical conductivity of materials such as silicon when mechanically stressed. In contrast to other on-chip position sensing methods, piezoresistive sensors offer a wide sensing bandwidth, small footprint, and simple readout circuits (see [19, Table III]). However, their implementation conventionally requires extra masking, conducting layers deposition, and doping. These additional steps are not typically available through standard MEMS fabrication processes, such as MUMPs [20], limiting the widespread implementation of this sensing mechanism. To address this problem, a few designs are proposed in the literature, where sensing flexures are used as the piezoresistive sensors [21]-[24]. In [21] the piezoresistive property of a uniformly doped poly-silicon beam is used for displacement sensing in a MEMS device with thermal actuators. The sensor shows a nonlinear response most likely due to the second-order piezoresistive effect in the bending load, nonlinear displacement dependency of mechanical stress in the sensing beam, and the thermal coupling from actuator to sensor [21], [22]. In [23] and [24], the bulk piezoresistivity of T-shaped flexures are used for vibration detection of the resonators fabricated in the standard SOI-MEMS processes over extremely small displacement ranges.

In [1], we briefly reported the design of a piezoresistive displacement sensing scheme for a 1-degree-offreedom (1DOF) MEMS nanopositioner. In the design, the entire structure of two tilted beam flexures are used for displacement sensing. Hence, no extra masking process is needed, and footprint of the sensor is practically zero. The flexures' design leads to a linear relationship between the stage displacement and the sensor output voltage, which 
compares positively with the nonlinear sensor reported in [21]. In addition, the achieved resolution enhances over a relatively large displacement range by implementing differential sensing and using single crystal silicon as the structural material [1], [17], [21].

In this paper, a comprehensive study is conducted on the design and modeling of the device reported in [1]. We develop an analytical model that provides insight into the geometrical design of the sensor and the effects of different factors on its performance. This model is also used to discuss the linearity of the sensor. The device is thoroughly characterized for a variety of biasing conditions both in time and frequency domains, and discussions are provided for the observed behaviors using the analytical model. The remainder of the paper continues as follows: In Sec. II the fabricated device and its working principle is presented. Sec. III details the theoretical model and the design aspects of the device. In Sec. IV and V, the static and dynamic characteristics of the sensor are investigated, respectively. The sensor feedthrough signal observed in measurements is studied by developing a model-based approach in Sec. V. Sec. VI discusses the noise and resolution of the sensor and sec. VII concludes the paper.

\section{Differential Piezoresistive SEnsor}

Fig. 1a shows the scanning electron microscope (SEM) image of the proposed piezoresistive displacement sensing structure implemented within a 1DOF MEMS nanopositioner. The nanopositioner comprises a stage that is suspended at the center of the device by mechanical flexures and actuated by electrostatic combs through the shuttle beams. Electrostatic actuators are implemented on both sides to move the stage bidirectionally. The comb structures are realized in the trapezoidal shape to reduce their mass while maintaining the required mechanical strength. As a part of the suspension system, four tilted beams are symmetrically implemented on both sides of the stage. A pair of the tilted flexure beams designated as $R_{p 1}$ and $R_{p 2}$ in Fig. 1a constitutes the piezoresistive elements employed in the sensor.

In contrast to the conventional MEMS piezoresistive sensors, no extra masking and doping steps are required. Hence, MEMSCAP's standard SOIMUMPs process was chosen for the fabrication [20]. Electrically conducting layers with a total thickness of $520 \mathrm{~nm}$, comprising gold and chromium, are deposited on the stage, the tilted beams on one side of the stage, and the shuttle beams. As shown in Fig. 1a, these gold plated flexures are connected to the electrical ground pads, establishing the earth connections for both actuation and sensing circuits.

As seen in Fig. 1b, dummy resistors are also fabricated on the same chip to be used as components of the readout circuit. These resistors have identical dimensions as the piezoresistive beams, and since they are fabricated elsewhere on the chip, they experience no mechanical stress during the device operation.

Fig. 2a schematically shows the operation principle of the sensor. In the nanopositioner, the rectilinear motion of the stage in either direction induces tensile and compressive

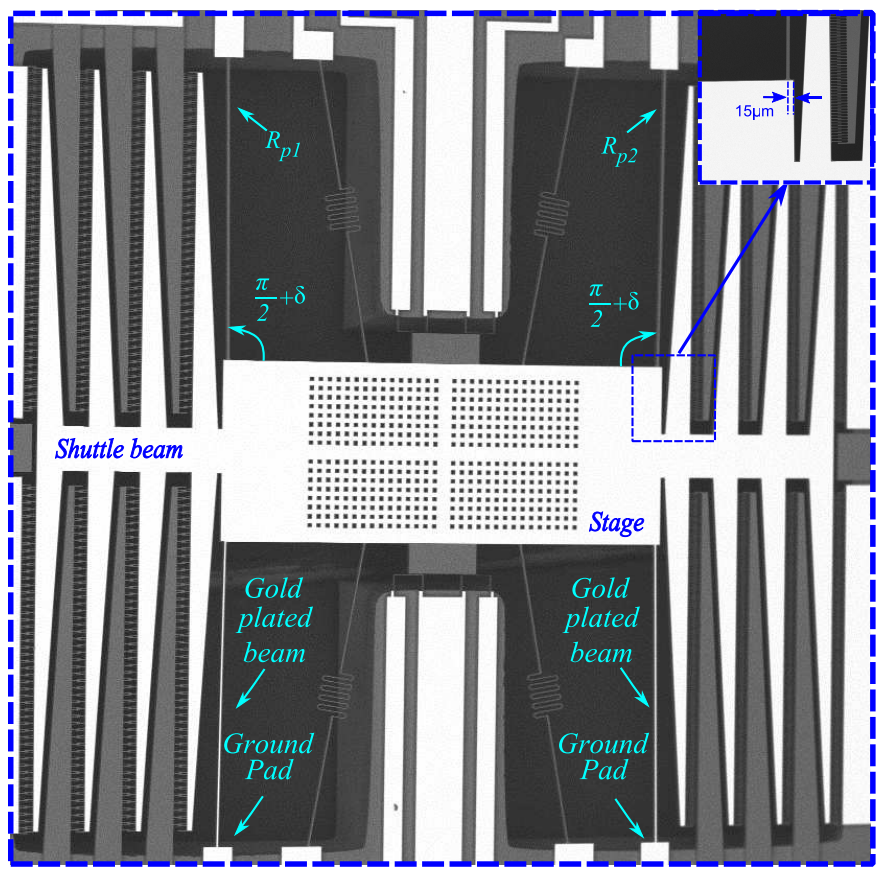

(a)

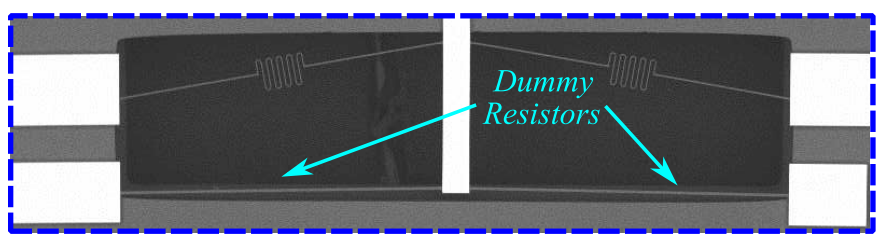

(b)

Fig. 1. (a) The SEM image of the piezoresistive sensing platform implemented as the suspension system in a 1DOF nanopositioning stage. Closed-ups show the inclination distance in the flexure. (b) Dummy resistors fabricated separately on the chip.

mechanical stresses along the tilted piezoresistive beams $\left(R_{p 1}\right.$ and $\left.R_{p 2}\right)$. These longitudinal stresses lead to opposite resistance variations in these beams due to the inherent piezoresistivity of silicon. These resistance variations are mapped to the sensor's output voltage, which is proportional to the stage displacement, using a Wheatstone bridge as the readout circuit. The Wheatstone bridge is schematically shown in Fig. 2b in a half bridge configuration. The dummy resistors designated as $R_{d 1}$ and $R_{d 2}$ are used to complete the Wheatstone bridge topology.

Fig. 2c illustrates the schematic of the actuation circuit, which is implemented independently of the readout circuit. An actuation voltage $\left(v_{a}\right)$ with opposite signs plus a constant dc voltage $\left(V_{q}\right)$ are applied to the opposing electrostatic combs on opposite sides to mitigate the inherent quadratic nonlinearity of the electrostatic actuator. The resulting force is proportional to $V_{q} \times v_{a}$ which is linear with respect to the actuation signal $\left(v_{a}\right)$ [11], [25]. During all experiments, a constant voltage of $30 \mathrm{~V}$ is used as $V_{q}$.

In Table I, the geometrical properties of the proposed nanopositioner are provided. An analytical model describing the tilted beam deflection and its axial force is presented next, based on which the sensor mechanical properties and its linearity are discussed. 


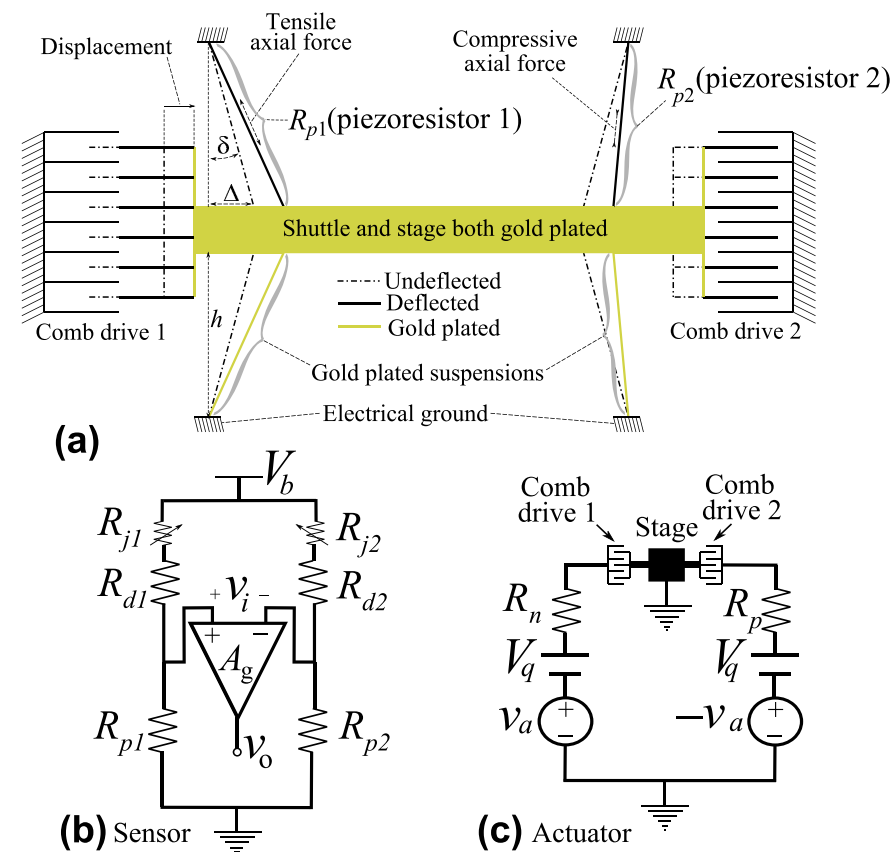

Fig. 2. (a) Schematics of the 1DOF nanopositioner with tilted flexures. In (b), the schematic of the readout circuit for the piezoresistive sensors is shown. The potentiometers $\left(\mathrm{R}_{\mathrm{j} 1}\right.$ and $\left.\mathrm{R}_{\mathrm{j} 2}\right)$ are incorporated for fine tuning of the Wheatstone bridge at zero displacement. (c) shows the actuation circuit configuration.

TABLE I

THE GeOMETRICAL AND MATERIAL PROPERTIES OF THE 1DOF NANOPOSITIONER

\begin{tabular}{|c|c|c|}
\hline \multicolumn{2}{|c|}{ Young modulus $E=169 \mathrm{GPa}$} & Thickness: $25 \mu \mathrm{m}$ \\
\hline \multirow{2}{*}{ Tilted beams } & Normal Length $h=1 \mathrm{~mm}$ & Width $w=10 \mu \mathrm{m}$ \\
\hline & \multicolumn{2}{|c|}{ Inclination distance, angle: $\Delta=15 \mu \mathrm{m}, \delta=0.86^{\circ}$} \\
\hline \multirow{2}{*}{ Comb finger } & Air gap: $2 \mu \mathrm{m}$ & Engagement: $12 \mu \mathrm{m}$ \\
\hline & Length: $28 \mu m$ & Width: $2 \mu \mathrm{m}$ \\
\hline Comb structures & \multicolumn{2}{|c|}{ Trapezoidal: Length:1175 $\mu \mathrm{m}$, Bases: $20,90 \mu \mathrm{m}$} \\
\hline Shuttle beams & \multicolumn{2}{|c|}{$613 \mu \mathrm{m} \times 150 \mu \mathrm{m}$} \\
\hline Stage & \multicolumn{2}{|c|}{$1500 \mu m \times 600 \mu m$} \\
\hline
\end{tabular}

\section{Analytical Modeling And Design}

As depicted in Fig. 2a, a pair of tilted beams undergoes compression while the opposite pair experiences tensile forces along their length. Regardless of the direction of the longitudinal force, the beams have the same boundary conditions: rigidly clamped at one end and a slider (guided) constraint at the other due to the structural symmetry. The analytical models describing a tilted beam's behavior under compressive and tensile conditions are separately derived and presented below.

\section{A. Compression}

When the stage moves toward a tilted beam, a compressive force is developed along the beam's length. In contrast to the conventional deflection theory for clamped-guided beams [26], in this case, the axial force and the change in the beam's length cannot be ignored. This loading condition for a tilted beam with the length of $(l)$ and tilting angle of $(\delta)$ is shown schematically in Fig 3. The horizontal displacement of the stage toward the anchor point is designated as $q$.

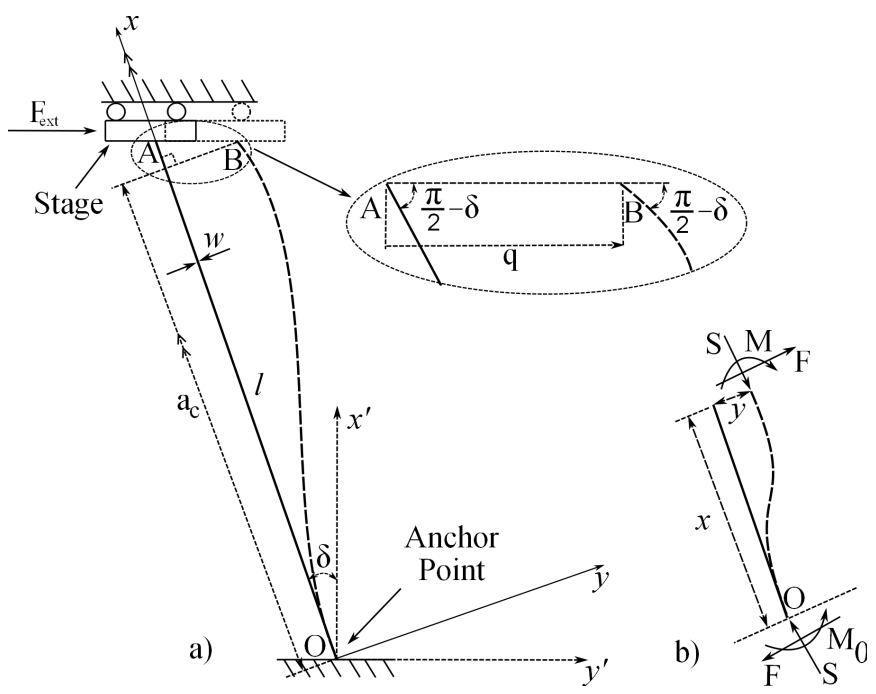

Fig. 3. (a) The schematic of the tilted beam experiencing compressive axial force due to the stage displacement toward the beam's anchor point. (b) The free body diagram of a beam segment.

To obtain a mathematical model of the flexure, a Cartesian coordinate is considered with its origin at the anchor point $(O)$ and the $x$ and $y$ axes directed along and perpendicular to the undeflected beam, respectively. Regarding the static equilibrium condition of the beam segment shown in Fig. 3b, we can write (1) for the beam's deflection profile $(y(x))$ using the Euler-Bernoulli theory.

$$
E I y^{\prime \prime}=M=M_{0}-S y-F x
$$

In (1), $y^{\prime \prime}$ denotes the second derivative of $(y)$ with respect to the length coordinate $(x)$, while $E$ and $I$ are Young's Modulus and second moment of inertia of the beam's cross section, respectively. $S$ is the constant axial force, $F$ is the transverse force along the beam, and the moment at the anchor point is designated by $M_{0}$. For the in-plane deflection of a beam with the width of $w$ and thickness of $t, I$ is [26]:

$$
I=\frac{w^{3} t}{12}
$$

The general solution of (1) comprises a homogenous part (first two terms in (3)) and a particular solution (i.e. $C x+D$ ):

$$
y=A \sin (\lambda x)+B \cos (\lambda x)+C x+D
$$

where, $\lambda$ is defined in (4) based on the axial force $(S)$.

$$
\lambda=\sqrt{\frac{S}{E I}}
$$

Inserting the particular solution in (1), we obtain $C$ and $D$ as:

$$
C=-\frac{F}{S} \text { and } D=\frac{M_{0}}{S}
$$

To obtain the remaining unknown parameters in (3), we may use the following boundary conditions:

$$
\begin{aligned}
y(x=0) & =0 \\
y^{\prime}(x=0) & =0 \\
y^{\prime}\left(x=a_{c}\right) & =0 \\
y\left(x=a_{c}\right) & =q \cos (\delta),
\end{aligned}
$$


where $a_{c}$ is the $x$ coordinate of the beam's guided end, and it can be calculated by (10) as a function of the stage displacement $(q)$.

$$
a_{c}=l-q \sin (\delta)
$$

It should be noted that in the small deflection condition $(q \ll l)$ or small inclination angle $(\delta), a_{c}$ is equal to $l$. Using (6) and (7), the unknown coefficients $A$ and $B$ can be obtained in terms of $M_{0}$ and $F$ as:

$$
A=\frac{F}{\lambda S} \text { and } B=\frac{-M_{0}}{S} \text {. }
$$

Substituting the unknown coefficients $(A, B, C, D)$ in (8), $M_{0}$ is calculated as a function of $F$ and $\lambda$ in (12).

$$
M_{0}=\frac{F\left[1-\cos \left(\lambda a_{c}\right)\right]}{\lambda \sin \left(\lambda a_{c}\right)} .
$$

Finally, by inserting (12) and (11) in (9), the force $(F)$ is obtained in terms of the axial force $(S)$ and $\lambda$ :

$$
F=\frac{S \lambda q \cos (\delta) \sin \left(\lambda a_{c}\right)}{X(\lambda)\left[1-\cos \left(\lambda a_{c}\right)\right]}
$$

where, $X(\lambda)$ is defined as:

$$
X(\lambda)=1-\cos \left(\lambda a_{c}\right)+\frac{\lambda \sin \left(\lambda a_{c}\right)}{1-\cos \left(\lambda a_{c}\right)}\left[\frac{\sin \left(\lambda a_{c}\right)}{\lambda}-a_{c}\right] .
$$

Since $\lambda$ is defined in terms of $S$ in (4), one extra condition is required to obtain a well defined solution for (3). Denoting the reduction in the length of the deflected beam by $\Delta l$, another boundary condition is obtained using Hooke's law [27]:

$$
S=\frac{A_{r} E}{l} \Delta l
$$

where $A_{r}$ is the the beam's cross section area. Based on the geometrical configuration, $\Delta l$ obeys the following equation:

$$
\Delta l=q \sin (\delta)-\frac{1}{2} \int_{0}^{a_{c}}\left(y^{\prime}\right)^{2} d x .
$$

The first term in (16) is defined based on the beam's length contraction due to the constrained displacement at its guided end. The second term estimates the length elongation of the deflected beam with the assumption $y^{\prime} \ll 1$ [26].

Although replacing (16) in (15) provides the required boundary condition, the implicit and nonlinear relation between the unknown parameters makes the derivation of a closed form solution for (3) problematic. Nevertheless, by substituting (13) in (12), $F$ and $M_{0}$ can be obtained as functions of the axial force $(S)$, and, as a result, the remaining parameters: $A, B, C, D$ are also obtained in terms of $S$ using (5) and (11). Hence, (15) and (16) can be written as follows:

$$
S=g(S)
$$

where, $g$ is a nonlinear function of $S$. For the tilted beams with the dimension stated in Table I and a guided end (stage) displacement of $q$, (17) is numerically solved using the fixedpoint iteration method [28]. In Fig. 4, the deflection profile of the tilted beam is presented for various stage displacements.

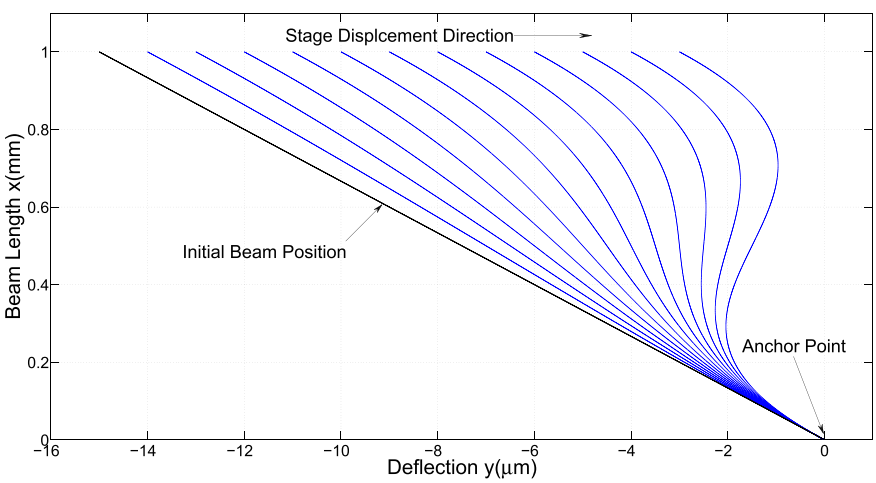

Fig. 4. The deflection profile of the tilted flexure beam while the stage moves toward the anchor point. Total stage displacement of $12 \mu \mathrm{m}$ is shown in incremental step size of $1 \mu \mathrm{m}$. The deflection is exaggerated by reporting it in $(\mu \mathrm{m})$, while the beam length is in $(\mathrm{mm})$.

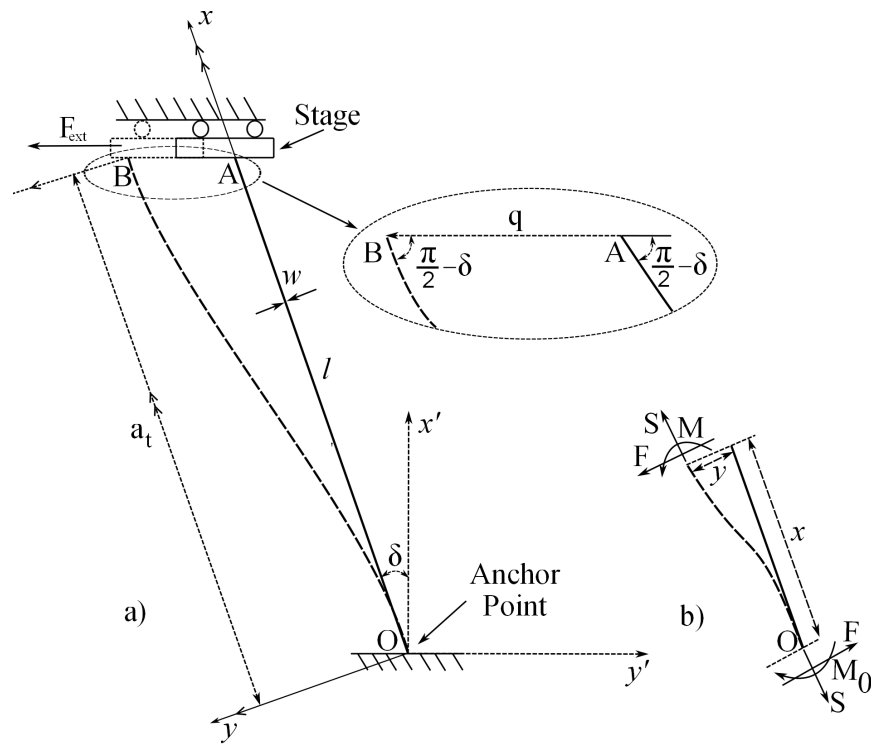

Fig. 5. (a) The schematic of the beam in tensile condition as the stage moves away from the anchor point. (b) The free body diagram of a beam segment.

Note that for the sake of clarity, the deflection profile of the beam is illustrated in the anchor point coordinate system $\left(x^{\prime} y^{\prime}\right.$ in Fig. 3) rather than the beam coordinate system ( $\left.x y\right)$. Clearly, the boundary conditions are satisfied in the beam's deflection profile for all stage displacements.

\section{B. Tension}

An analytical model is also derived for the tilted beam with the same boundary conditions when the stage moves horizontally away from the anchor point. In this case, as shown schematically in Fig. 5, the axial force $(S)$ is tensile. Considering the Euler-Bernoulli beam theory and the free body diagram depicted in Fig. 5b, the beam's deflection profile $(y(x))$ satisfies the following equation:

$$
E I y^{\prime \prime}=M_{0}+S y-F x .
$$

Similar to (1), the complete form of solution for the ordinary differential equation (18) is:

$$
y(x)=A \cosh (\lambda x)+B \sinh (\lambda x)+C x+D .
$$


Using the same approach explained in Sec. (III-A), $C$ and $D$ are obtained as follows:

$$
C=\frac{F}{S} \text { and } D=\frac{-M_{0}}{S} \text {. }
$$

The boundary conditions for the compressive mode presented in (6) and (7) are still valid leading to the following values for $\mathrm{A}$ and $\mathrm{B}$.

$$
A=\frac{M_{0}}{S} \text { and } B=\frac{-F}{\lambda S}
$$

However, in (8) and (9), $a_{c}$ should be replaced by $a_{t}$ defined below due to the increase in the beam's length.

$$
a_{t}=l+q \sin (\delta)
$$

Using these two boundary conditions, $M_{0}$ and $F$ are obtained as:

$$
\begin{aligned}
M_{0} & =\frac{F\left[\cosh \left(\lambda a_{t}\right)-1\right]}{\lambda \sinh \left(\lambda a_{t}\right)} \\
F & =\frac{S \lambda q \cos (\delta) \sinh \left(\lambda a_{t}\right)}{X(\lambda)\left[\cosh \left(\lambda a_{t}\right)-1\right]}
\end{aligned}
$$

where $X(\lambda)$ is defined as:

$$
X(\lambda)=\frac{\lambda \sinh \left(\lambda a_{t}\right)}{\cosh \left(\lambda a_{t}\right)-1}\left[a_{t}-\frac{\sinh \left(\lambda a_{t}\right)}{\lambda}\right]+\cosh \left(\lambda a_{t}\right)-1
$$

Similar to the compression condition, by substituting (24) in (23) and (21) all unknowns can be defined in terms of $S$.

The tensile axial force can be similarly obtained based on the Hooke's law as presented in (15) provided that the increase in the length $\left(\Delta_{t} l\right)$ which is defined in (26) is considered.

$$
\Delta_{t} l=q \sin (\delta)+\frac{1}{2} \int_{0}^{a_{t}}\left(y^{\prime}\right)^{2} d x
$$

Numerical method is also used to obtain the deflection profiles of the beam for different stage displacements, which are plotted in Fig. 6.

\section{Mechanical Design}

Mechanical properties such as stiffness and the allowable displacement range of the tilted beams as the suspension system are investigated here based on the analytical model obtained in previous section. As shown in Fig. 3, the external force required to move the stage toward the anchor point can be written as (27).

$$
F_{\text {ext }}=F \cos (\delta)+S \sin (\delta)
$$

This force is calculated and shown in Fig. 7 for a displacement range of $20 \mu \mathrm{m}$. The beam demonstrates a softening behavior with the displacement. When the slider moves about $10 \mu \mathrm{m}$, the stiffness of the beam reduces to zero. Afterward, until the beam's end passes the horizontal position of the anchor point, the beam shows a negative stiffness characteristic where a further increase in displacement requires a decrease in the external force [29]. After the slider passes the anchor point, the beam again shows positive stiffness. We designate the values of the external force and the

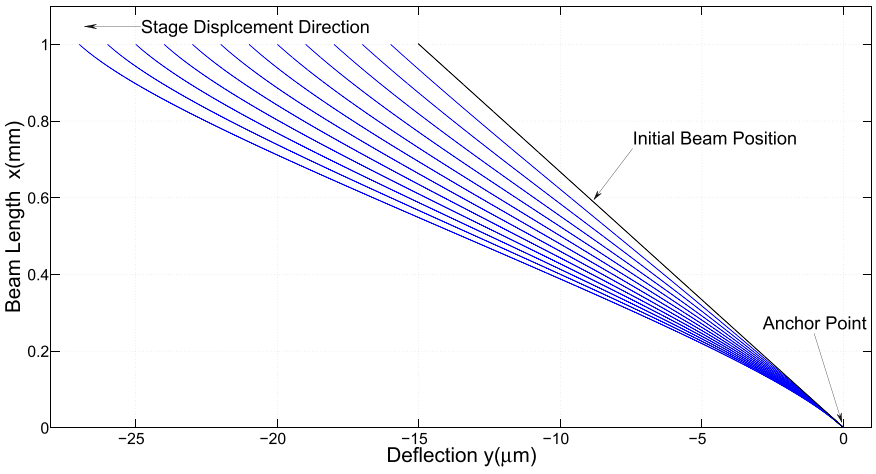

Fig. 6. The deflection of the beam under tensile loading condition. Here the stage is moving away from the anchor point. The profiles are provided for the maximum stage displacement of $12 \mu \mathrm{m}$ with the incremental step of $1 \mu \mathrm{m}$. To have a clear illustration, the deflection is exaggerated by reporting it in $(\mu \mathrm{m})$, while the beam length is in $(\mathrm{mm})$.

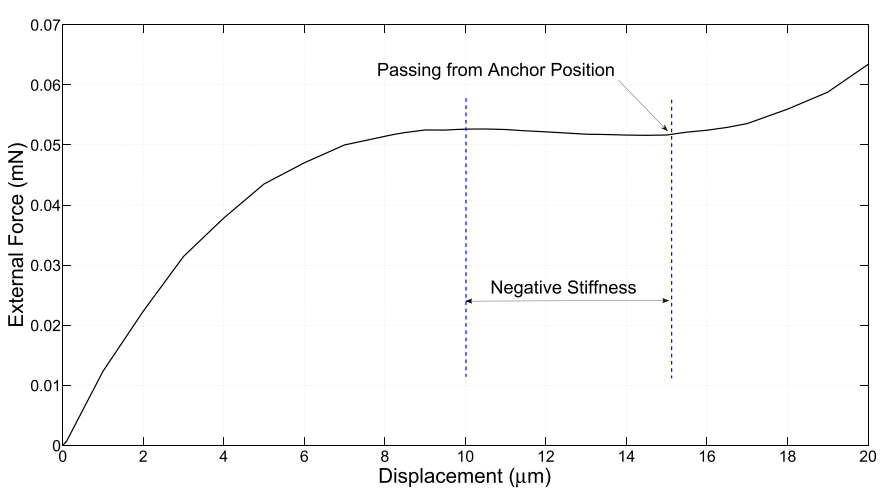

Fig. 7. The external force versus slider displacement when the beam moves toward the anchor point (compression state). After $10 \mu \mathrm{m}$ displacement the beam shows a negative stiffness, and when its end passes the horizontal position of the anchor point, it shows a positive stiffness again.

displacement (about $10 \mu \mathrm{m}$ ) beyond which the beam shows the first zero stiffness as the critical force and critical displacement, respectively [30]. Note that these critical values may vary if structural and material imperfections exist in the beam.

Regarding Fig. 5, (27) is also valid for the beam in tension. The external force is also presented in Fig. 8 as a function of the beam's guided end displacement for tensile condition. In comparison with the compressed beam, a hardening behavior is demonstrated while the beam's guided end moves away from anchor point. The hardening behavior is expected as reported in [27] for the straight clamped-guided beam with tensile axial force. In the same figure, the force-displacement characteristics of the nanopositioner's suspension system is also provided. The suspension system comprises four beams: a compressive pair and a tensile pair. In comparison with Fig. 7, the beams in the tensile conditions are stiffer when undergone large deflections, so they mostly contribute to the total suspension system stiffness. In a large displacement range (above $10 \mu \mathrm{m}$ ), therefore, a hardening behavior is expected for the entire suspension system.

Regarding (16) and (26), for a specific displacement, the developed axial force $(S)$ increases with the initial inclination distance ( $\Delta$ in Fig. 2a). As explained in the next section, 


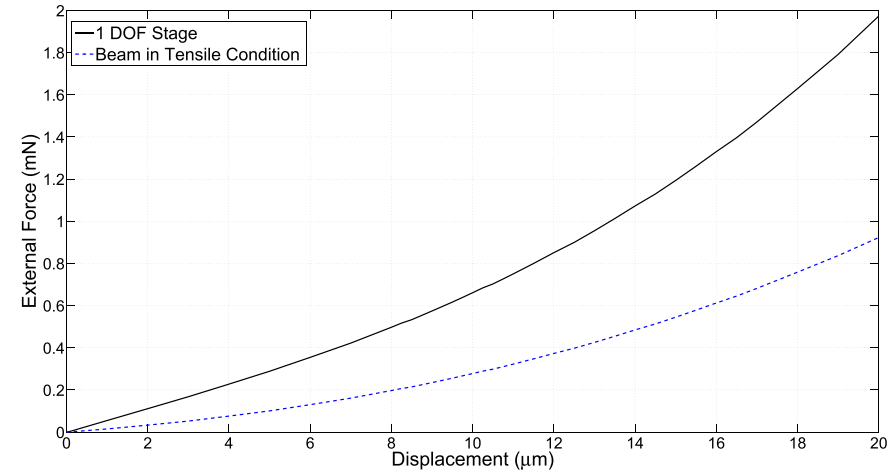

Fig. 8. The external force versus the beam's tip displacement. The force-displacement behavior of the 1DOF nanopositioner is also shown.

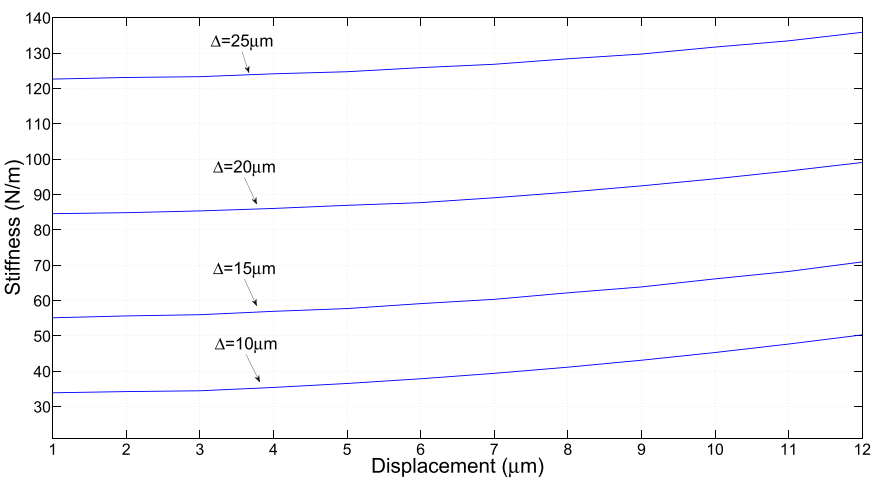

Fig. 9. The stiffness variation of the suspension system in various inclination distances $(\Delta)$ versus the stage displacement. The stiffness increases nonlinearly with the tilting angle.

this increase in the axial forces leads to an enhancement of the sensor sensitivity. However, the stiffness of the tilted beams changes drastically with variation of $\Delta$. Keeping a constant normal distance between the stage and the beam anchor i.e. $h=1 \mathrm{~mm}$, the stiffness of the suspension system, is depicted in Fig. 9 for different inclination distances $(\Delta)$ versus the stage displacements. The geometrical properties are considered as reported in Table I. Clearly, the stiffness increases nonlinearly with the inclination distance which ultimately leads to a decrease in the achievable displacement range of the nanopositioner.

As another design criterion, the maximum normal stress in beam's cross section is presented in (28) for both tensile and compression cases.

$$
\sigma_{\max }=\frac{S}{A_{r}}+\frac{M_{\max } w}{2 I}
$$

The second term in (28) is the component of the normal stress induced by the maximum moment $\left(M_{\max }\right)$ along the beam. Over a displacement range of $\pm 10 \mu \mathrm{m}$, the normal stress is calculated as $104 \mathrm{MPa}$ and $55 \mathrm{MPa}$ for tensile and compression conditions, respectively. Based on the silicon yield stress of $1.97 \mathrm{GPa}$ with Weibull modulus of 8.9 [31], the beams are designed to work in a safe margin.

\section{Sensor Linearity}

Using the analytical model, the linearity of this sensing platform with respect to the stage displacement can be evaluated. In the standard SOIMUMPs process, an n-type SOI wafer is typically used. In this process, the doping concentration on the top surface of the entire wafer is further enhanced by additional doping steps using a phosphosilicate glass (PSG) layer [20]. The doping concentration tends to be non-uniform along the thickness, but its profile in this direction is almost the same over the entire wafer surface. Thus, piezoresistive beams have almost identical doping profile along their thicknesses.

In addition to the doping level, the piezoresistive effect also depends on the electrical current and the crystallographic direction [32]. Since both beams are implemented in a parallel configuration (ignoring the small tilting angle of $0.86^{\circ}$ ), the piezoresistive coefficients are almost identical for both beams. Assuming $R_{p 1}$ and $R_{p 2}$ are respectively experiencing the compressive $\left(S_{p}\right)$ and tensile $\left(S_{t}\right)$ axial forces, we may express their actual values under the stresses as follows:

$$
\begin{aligned}
& R_{p 1}^{\prime}=R_{p 1}\left(1+\sigma_{p}\left|\pi_{l}\right|\right), \sigma_{p}=\frac{S_{p}}{A_{r}} \\
& R_{p 2}^{\prime}=R_{p 2}\left(1-\sigma_{t}\left|\pi_{l}\right|\right), \sigma_{t}=\frac{S_{t}}{A_{r}}
\end{aligned}
$$

where $\pi_{l}$ is the effective piezoresistive coefficient along the beams associated with the n-type silicon layer. Since the Wheatstone bridge in Fig. $2 \mathrm{~b}$ is balanced, by tuning the potentiometers whilst the stage is at its equilibrium (zero stresses), we have $R_{2} \times R_{p 1}=R_{1} \times R_{p 2}$ where $R_{2}=R_{d 2}+R_{j 2}$ and $R_{1}=R_{d 1}+R_{j 1}$ with $R_{j 1}$ and $R_{j 2}$ referring to the tuned values of the potentiometers. For small resistance variations, the sensor output can be written as:

$$
V_{\text {out }}=V_{b} A_{g}\left|\pi_{l}\right| \frac{R_{2} R_{p 1}\left(\sigma_{p}+\sigma_{t}\right)}{\left(R_{1}+R_{p 1}\right)\left(R_{2}+R_{p 2}\right)}
$$

where $A_{g}$ is the gain of the differential amplifier (refer to Fig. 2b). Practically, the assumption of $R_{p 1} \simeq R_{p 2} \simeq R_{1} \simeq R_{2}$ is valid due to the identical dimensions of piezo- and dummy resistors, hence, (31) can be simplified as:

$$
V_{\text {out }}=V_{b} A_{g} \frac{\left|\pi_{l}\right|}{4}\left(\sigma_{p}+\sigma_{t}\right)=\frac{V_{b} A_{g}\left|\pi_{l}\right|}{4 A_{r}}\left(S_{p}+S_{t}\right) .
$$

Clearly in (32), the output voltage of the sensor is proportional to the sum of the compressive and tensile forces in the tilted beams i.e. $\left(S_{p}+S_{t}\right)$. These axial forces and their sum are obtained analytically and depicted in Fig. 10 as a function of the stage displacement. In contrast to the nonlinear profile of the individual forces, their addition shows a highly linear relation with respect to the stage displacement. Hence, the linearity of the displacement sensing is expected to be improved considerably using the proposed differential sensing approach.

\section{Piezoresistive Sensor: Static Behavior}

In this section, we investigate the static response of the sensor over a large displacement range. During the experiment, the actuators were dc biased at $30 \mathrm{~V}$ and a slow $(1 \mathrm{~Hz})$ 


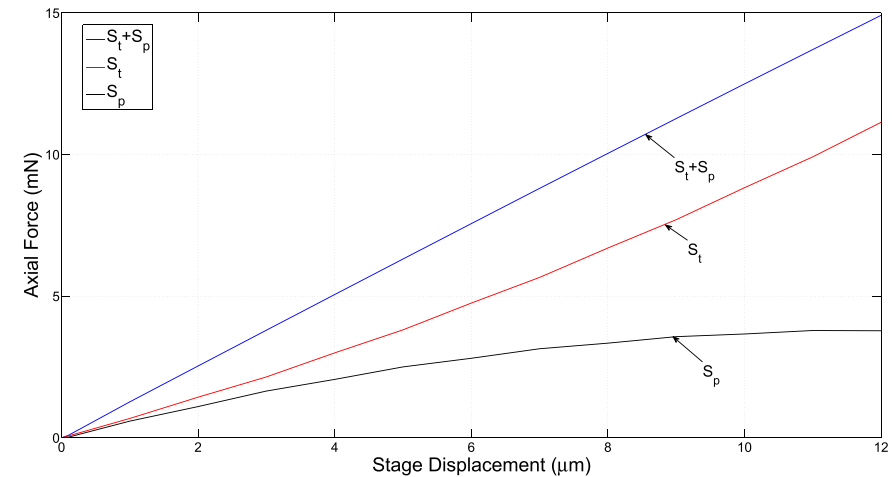

Fig. 10. The modeled axial force in compressive and tensile conditions in the tilted beams and their summation versus stage displacement.

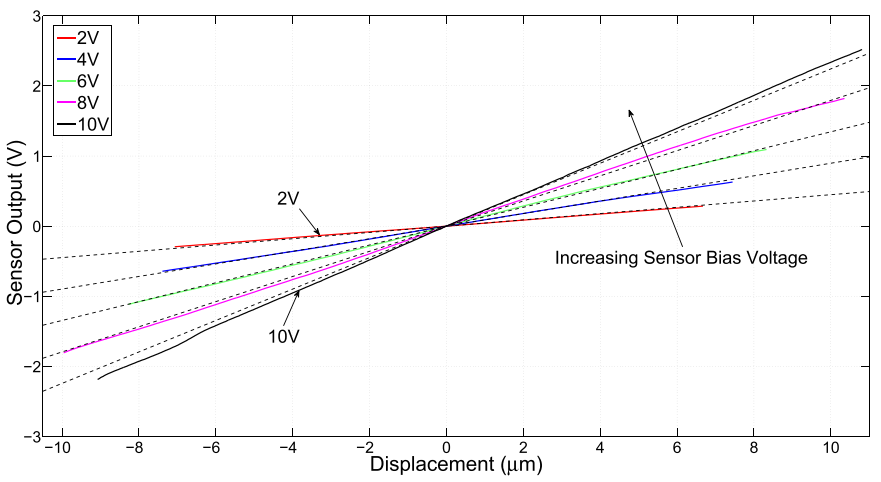

Fig. 11. The piezoresistive sensor output with different bias voltages versus displacement. The curves with dotted line (- -) show the theoretical results for each voltage.

triangular signal was applied differentially to them. The stage displacement was directly measured using Polytec MSA-050-3D Micro System Analyzer (MSA) and the sensor output was recorded simultaneously. The experiments were carried out for the sensor with different voltage biases $\left(V_{b}\right.$ in Fig. $2 \mathrm{~b}$ ) ranging from $2 \mathrm{~V}$ to $10 \mathrm{~V}$. Fig 11 shows the piezoresistive sensor output versus stage displacement for different bias voltages. Clearly, the sensor operates linearly in the displacement range of the stage as expected from the theoretical model.

Using (32) and the experimental data, the average value of $\pi_{l}$ is obtained as $-16.69 \times 10^{-11} \mathrm{~Pa}^{-1}$. In Fig. 11 the analytical results are presented as dotted lines. Experimental and analytical results are in close agreement. The slight deviation between the experimental and analytical model can be attributed to the temperature elevation in the beams and dummy resistors and the assumption of invariant piezoresistive coefficient with respect to the bias voltage.

Introducing the calibration factor as the ratio of the sensor output voltage to the stage displacement, Fig. 12 illustrates its experimental variation with bias voltage during both falling and rising edges of the actuation signal. Clearly, the calibration factor changes linearly with the sensor bias voltage and is identical for both rising and falling intervals, indicating no hysteresis effect during displacement sensing.

However, as the sensor bias voltage increases, the displacement-actuation profile of the stage deviates further

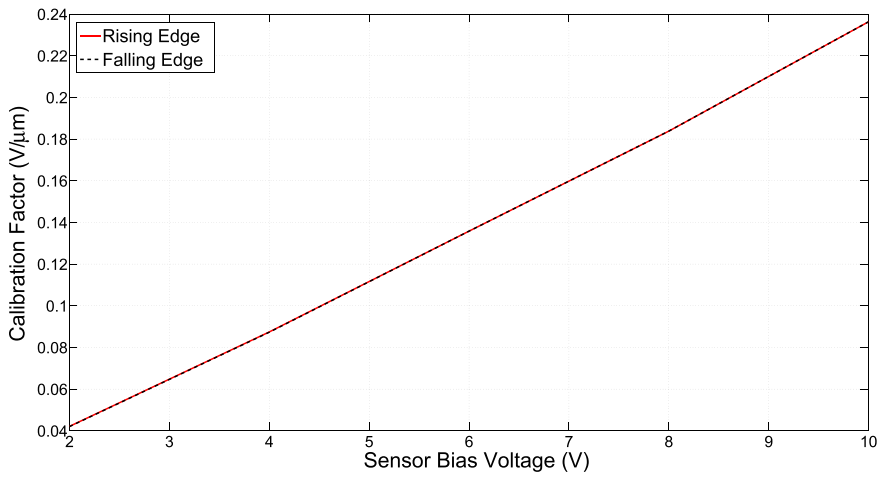

Fig. 12. The variation of the calibration factor in different sensor bias voltages for both rising and falling edges.

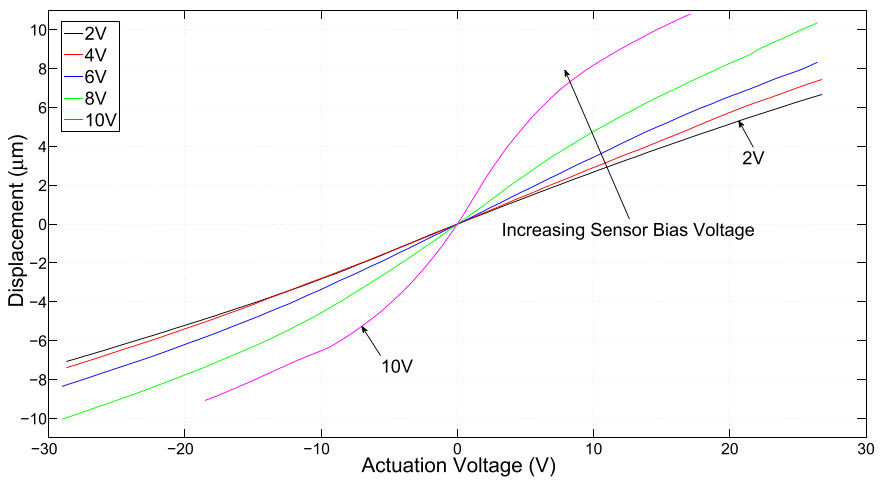

Fig. 13. The displacement of the stage versus actuation voltage in different bias voltages of the piezoresistive sensors obtained by the MSA.

from the linear, and the suspension system becomes more compliant as shown in Fig. 13. More compliant characteristics initially can be justified by the fact that the stiffness of the structural material decreases with temperature elevation, which occurs due to increase in Joule heating at higher bias voltages [33]. However, this effect may be insignificant due to the small temperature coefficient of the Young's modulus for silicon which is about $0.01 \% /{ }^{\circ} \mathrm{C}$. On the other hand, the thermal expansion of the beams induces an additional compressive axial force along both tilted beams. Considering this additional force in the analytical analysis reveals that the compliance of both beams increases with their thermal expansion. ${ }^{1}$ Hence, this effect seems to be dominant for the observed softening behavior. Note that, the thermal compressive force also decreases the critical values explained in Sec. III-A, and by applying a sufficiently large bias voltage, the beams will buckle at their equilibrium position.

Since the beams in tension provide the dominant contribution to the total stiffness, their characteristics are further investigated in the presence of the thermal stress.

\footnotetext{
${ }^{1}$ This is estimated by assuming an identical temperature increase for both beams. For the beam in compression, a length increase of $\Delta l_{t h}$ is added to (16) due to the thermal expansion. Since the tensile beam is also experiencing an axial compressive stress at sufficiently small displacements, the equations in Sec.III-A are applicable here provided that (22) is used to define $a_{c}$ and (16) is replaced by $\Delta l=\Delta l_{t h}-\Delta_{t} l$. Calculating the exact thermal expansion requires the modeling of the temperature distribution along the beam which is beyond the scope of this paper.
} 
Indeed, this compressive stress causes all beams to operate in the post-buckling range at high sensor bias voltages such as $10 \mathrm{~V}$ within a limited displacement range. Thus, in Fig. 13, the beams show a higher compliance for the sensor bias of $10 \mathrm{~V}$ and less than $10 \mu \mathrm{m}$ displacement range. However, the post-buckling condition only continues up to a displacement range where the beams develop enough tensile axial force to overcome the thermal compressive stress. For larger displacements though, as shown in Fig. 13, the beams, and consequently the suspension system, behave stiffer again by developing tensile axial forces. In addition, the post-buckling condition of the beams and presence of structural asymmetries lead to the asymmetric displacement of the stage around its rest position, which is observable for $V_{b}=10 \mathrm{~V}$ in Fig. 13 .

Based on the static test results, the sensor illustrates more linear structural behavior with bias voltages below $10 \mathrm{~V}$. However, inevitable phenomena such as intrinsic fabrication stress in the beams, geometrical tolerances, and compliance of the anchor points may practically change the achievable displacement range and affect the suitable selection of sensor bias voltage.

By comparing Figs. 11 and 13, we note that although the thermal stress leads to a softening behavior of the stage at the higher bias voltages, the sensor is still operating linearly. This is expected due to the fact that thermo-mechanical stresses induced either by the ambient temperature variation or the increase of the bias voltage are almost identical for piezo- and dummy resistors. Therefore, due to the Wheatstone bridge configuration of these resistors, their identical variations will be canceled out at the sensor output.

The Wheatstone bridge, however, cannot cancel the temperature dependency of the piezoresistive coefficient $\left(\pi_{l}\right)$. Nevertheless, this sensor will operate linearly for different doping concentrations and profiles as long as they are identical for the beams. The temperature dependency of the piezoresistive coefficient, therefore, can be alleviated by incorporating a higher level of the doping concentration [32]. In addition, extra compensation techniques may also be implemented in the design and/or in the readout circuitry to mitigate the remaining effects of the ambient temperature variations and fabrication process tolerances [34], [35].

\section{Piezoresistive Sensor: Dynamic Behavior}

Before examining the frequency response of the sensor, the frequency response of the stage was obtained by applying a band limited chirp signal to the linear actuation system described in Sec. II. The displacement of the stage was measured by the MSA and its Bode plot is presented in Fig. 14. The softening behavior of the suspension is also visible in the frequency domain at large sensor bias values. When the sensor is inactive $\left(V_{b}=0 \mathrm{~V}\right)$, the stage features a dominant resonance frequency at about $2870 \mathrm{~Hz}$, which is reduced to about $1560 \mathrm{~Hz}$ at $10 \mathrm{~V}$. Moreover, the low frequency gain of the device increases with the sensor bias voltage, further confirming the suspension softening process.

The $\mathrm{Q}$ factor, which indicates of the sharpness of the resonance peak and the amount of energy dissipation,

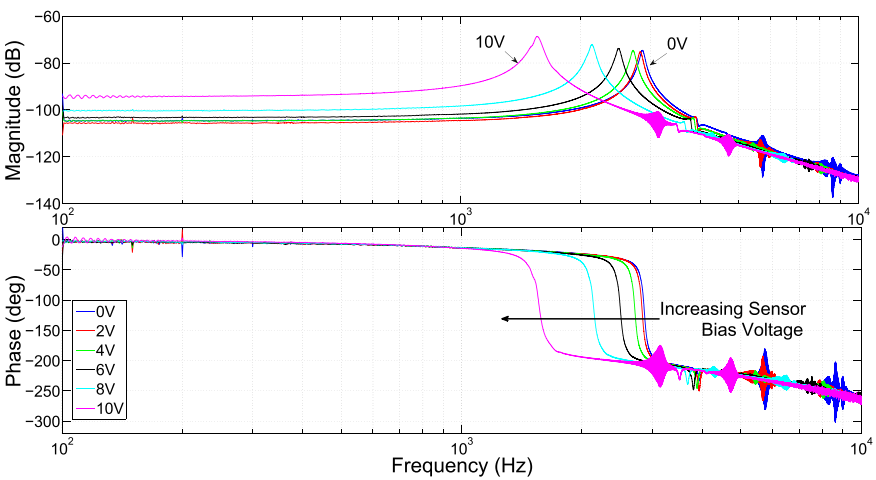

Fig. 14. Frequency response obtained using the MSA in different bias voltages of the piezoresistive sensor. The magnitude value is defined based on $1 \mathrm{~m}$ of displacement i.e.: $0 \mathrm{~d} B=1 \mathrm{~m}$.

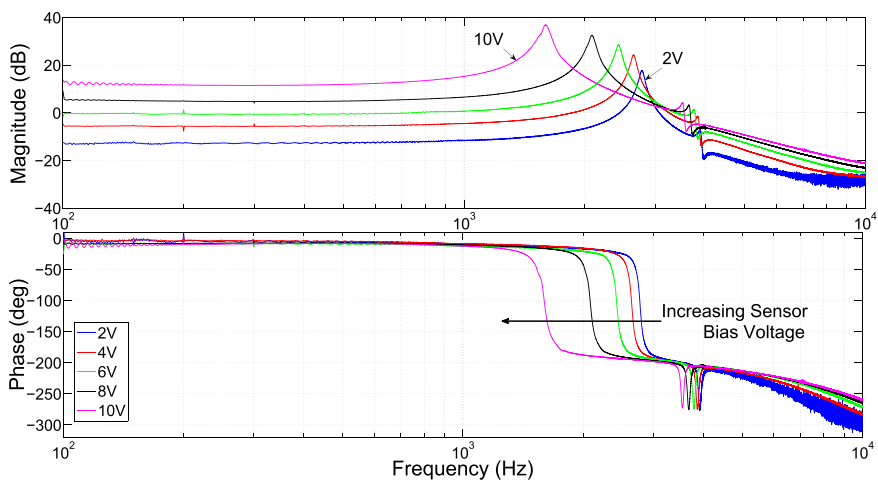

Fig. 15. Piezoresistive sensor output at different sensor bias voltages.

is calculated [36]. In the frequency response illustrated in Fig. 14, the $\mathrm{Q}$ factor is reduced from about 36 for the stage with sensor being inactive to 22 when $10 \mathrm{~V}$ bias voltage is applied to the sensor. The $\mathrm{Q}$ factor reduction is a sign of the increase in the energy dissipation, which can be explained by the thermoelastic damping effect in the beams that increases with the temperature [37].

The sensor's frequency response was also obtained while employing the same test conditions, and is shown in Fig. 15 for various bias voltages. Clearly, the sensor output closely matches the dynamic response of the stage displacement for all bias voltages up to approximately twice the stage's resonant frequency. However, the sensor shows a high-pass behavior due to the feedthrough signal from the electrostatic actuators [1]. This limits the sensor bandwidth, which is further detailed in the following section.

\section{A. Sensor Feedthrough}

To understand the origin of the observed feedthrough, the actuation voltage $\left(V_{q}\right)$ is set to $0 \mathrm{~V}$ (switched off) in the experiments. Due to the structural symmetry, the net actuating force experienced by the stage is practically zero. Hence, the stage remains almost stationary irrespective of the differential actuation signal $\left(v_{a}(t)\right)$, and its dynamics has a negligible effect on the sensor output. In Fig. 16, the feedthrough signal measured by employing this test is compared with the nanopositioner's frequency response obtained by the MSA and 


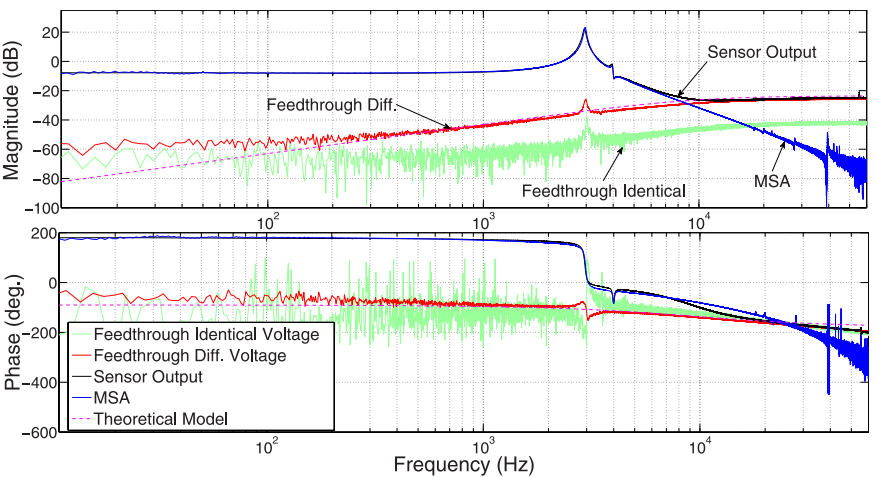

Fig. 16. The sensor output in different actuation conditions compared with the MSA measurement. The small peak in the feedthrough signal originates from slight asymmetric structures of the electrostatic actuators in each side.

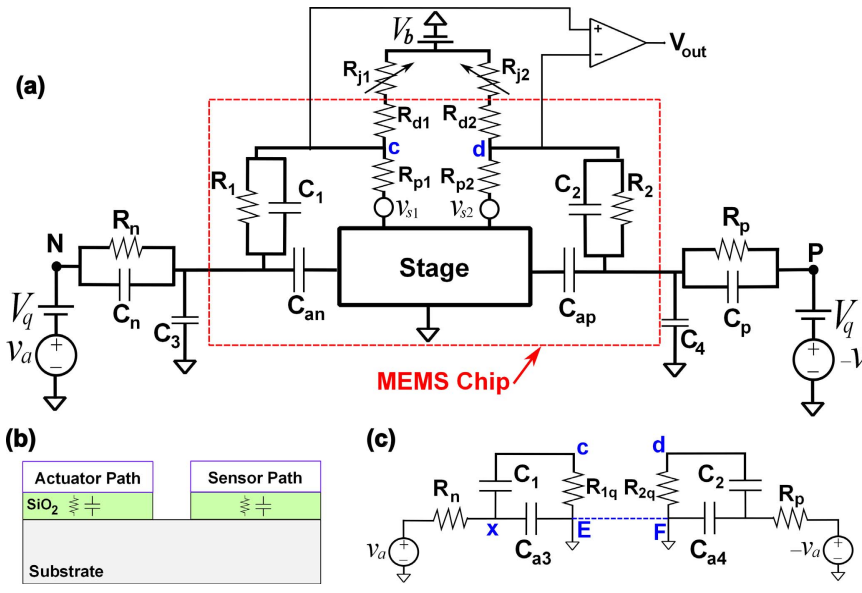

Fig. 17. (a) the schematic of the electrical lumped model for the sensing and actuating circuit.(b) Two adjacent sensor and actuator paths fabricated on the device layer and insulated by the oxide layer. (c) A simplified electrical model of the sensing and actuating of the MEMS device.

the sensor with the bias voltage of $4 \mathrm{~V}$. Clearly, the sensor and the MSA measurements are consistent up to about $8 \mathrm{kHz}$, after which the feedthrough signal becomes dominant at the sensor output.

As an alternative approach, we applied identical actuation signals $\left(v_{a}\right)$ with similar polarities to the comb actuators on both sides, simultaneously. Also in this condition, the net actuating force on the stage is negligible [38]. As seen in Fig. 16, the feedthrough signal in this condition is drastically mitigated compared with the differential actuation test. This result confirms that the crosstalk effect in the device is mainly due to the differential actuation and sensing configurations, and, regarding the nanopositioner's symmetry, the parasitic elements on each side are almost identical.

In order to investigate the observed crosstalk, an electrical model of the MEMS device including the sensing and the actuating circuits is presented in Fig. 17a. The rectangular frame with dotted lines indicates the physical boundaries of the MEMS chip. As a general case, unequal values for corresponding elements are assumed on either side of the circuit. The external resistors $R_{p}$ and $R_{n}$ are implemented in the actuation path to limit the short circuit current should snap-in occur [38]. $C_{a n}$ refers to the capacitance of the left comb drive plus any parasitic elements between the left actuation and the ground paths on the chip, and $C_{a p}$ is similarly defined for the right side. The capacitance between the PCB tracks and the electrical ground is modeled as $C_{3}$ and $C_{4}$. Furthermore, the voltage sources: $v_{s 1}$ and $v_{s 2}$ represent the change in the resistance of the piezoresistors due to the stage movement.

Sensing and actuation tracks in the MEMS chip are insulated by a buried oxide $\left(\mathrm{SiO} \mathrm{O}_{2}\right)$ layer with the thickness of 2 microns. The parasitic elements between these electrical tracks connected via substrate are modeled as a parallel capacitor and resistor as shown schematically in Fig. 17b. These elements are also considered on each side of the circuit model as $R_{1}, C_{1}$ and $R_{2}, C_{2}$ in Fig. 17a.

The model is simplified and presented in Fig. 17c, where due to the high electrical resistance of $\mathrm{Si} O_{2}$ layer, $R_{1}$ and $R_{2}$ are assumed to be open circuited. The impedance of the capacitors associated with the external resistors $\left(C_{n}, C_{p}\right)$ is also assumed to be large in the frequency range of interest. In addition, the input impedance of the differential amplifier is considered to be infinite with a bandwidth larger than $100 \mathrm{kHz}$ [39].

During the feedthrough test, the stage displacement is negligible, hence: $v_{s 1,2}=0$. In the equivalent circuit shown in Fig. $17 \mathrm{c}, R_{1 q}$ and $R_{2 q}$ are:

$$
R_{1 q / 2 q}=\left(R_{j 1 / j 2}+R_{d 1 / d 2}\right) \| R_{p 1 / p 2} .
$$

The transfer function of the feedthrough signal from the actuation source $\left(v_{a}\right)$ to the amplifier input $\left(v_{c}\right)$ is presented as:

$$
F_{c a}(s)=\frac{s R_{1 q} C_{1}}{s^{2} C_{a 3} C_{1} R_{n} R_{1 q}+s\left[R_{n}\left(C_{a 3}+C_{1}\right)+R_{1 q} C_{1}\right]+1}
$$

where $C_{a 3}$ is the parallel combination of $C_{3}$ and $C_{a n}$. Similarly, the transfer function from the actuation signal $\left(-v_{a}\right)$ to $v_{d}$ is:

$$
F_{d a}(s)=\frac{s R_{2 q} C_{2}}{s^{2} C_{a 4} C_{2} R_{p} R_{2 q}+s\left[R_{p}\left(C_{a 4}+C_{2}\right)+R_{2 q} C_{2}\right]+1}
$$

where, $C_{a 4}$ is the parallel combination of $C_{a p}$ and $C_{4}$. Finally, the transfer function of the sensor output $\left(v_{\text {out }}\right)$ from the actuation signal $\left(v_{a}\right)$ is as follows:

$$
F_{\text {out }}(s)=A_{g}\left[F_{c a}(s)+F_{d a}(s)\right] .
$$

As expected, both transfer functions in (34) and (35) exhibit a zero at the origin. The measured value of the electrical resistance of the piezoresistors $\left(R_{p 1}\right.$ and $\left.R_{p 2}\right)$ are about $526 \Omega$. Employing E4980A precision LCR meter, about $16 \mathrm{pF}$, is measured for $C_{a n}$ and $C_{a p}$ and $13 \mathrm{pF}$ for $C_{3}$ and $C_{4}$. Using a dielectric constant of 3.9 for $\mathrm{SiO}_{2}$ [40], a capacitance of about $1 \mathrm{pF}$ is estimated for $C_{1}$ and $C_{2}$. In Fig. 16, the Bode plot for the transfer function of the sensor output $\left(F_{\text {out }}\right)$ with $R_{n, p}=566 \mathrm{k} \Omega$ is compared with the experiment results. The analytical model shows an acceptable consistency with the experimental feedthrough data with differential actuation signals. The model can also explain the reduction of the 


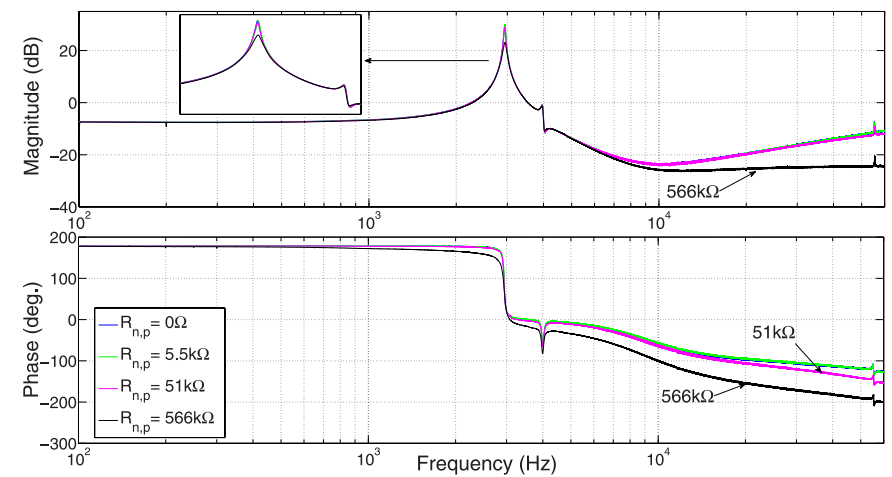

Fig. 18. The sensor output in frequency domain in different external resistors The results are compared with the sensor output without external resistor. In the closed-up view, the reduction in the magnitude of the resonant peak using a $566 \mathrm{k} \Omega$ external resistor is shown.

TABLE II

The Calibration Factor and Resolution of the Piezoresistive SENSOR IN VARIOUs Bias VolTages

\begin{tabular}{|c|c|c|c|c|c|}
\hline Bias $(\mathrm{V})$ & 2 & 4 & 6 & 8 & 10 \\
\hline \hline $\begin{array}{c}\text { Calibration } \\
\text { Factor }(V / \mu \mathrm{m})\end{array}$ & 0.0420 & 0.0873 & 0.1358 & 0.1838 & 0.2363 \\
\hline Resolution $(\mathrm{nm})$ & 2.2 & 1.4 & 0.9 & 1.7 & 3.7 \\
\hline
\end{tabular}

feedthrough in the second test, with identical actuation voltages. The model predicts an ideally zero feedthrough for a perfectly symmetrical device. The weak residual feedthrough observed in the experiments is due to the structural asymmetries arising from fabrication tolerances.

The analytical model provides insight into the origin of the feedthrough. Both transfer functions $\left(F_{c a}, F_{d a}\right)$ have a zero at the origin and two poles on the real axis, which lead to a bandpass behavior. The location of the poles can be changed along the real axis by changing the external resistors $\left(R_{n, p}\right)$. As these resistors increase, the distance between the poles also increases while the slow pole will move toward the origin and vice-versa. Hence, the feedthrough behavior of the sensor can be tuned to some extent by changing these resistors. This is experimentally demonstrated by obtaining frequency response of the system for various external resistors as shown in Fig. 18.

Although the implementation of these resistors can reduce the feedthrough effect, it will induce delays in the stage dynamics as seen in Fig. 18 for $R_{n, p}=566 \mathrm{k} \Omega$. It is straightforward to determine the transfer function from $v_{a}$ to $v_{x}$ using the proposed model and investigate the resistors' effect on the stage's dynamics.

\section{NOISE AND RESOlUtion}

The sensor noise is recorded in the time domain with the sampling frequency of $25.6 \mathrm{kHz}$, while all electrostatic actuators and the stage are connected to electrical ground. In Table II, the sensor resolution is presented for various bias voltages. In each bias, the resolution is obtained by converting the standard deviation of the noise signal to displacement. As seen in Table II, a resolution better than $4 \mathrm{~nm}$

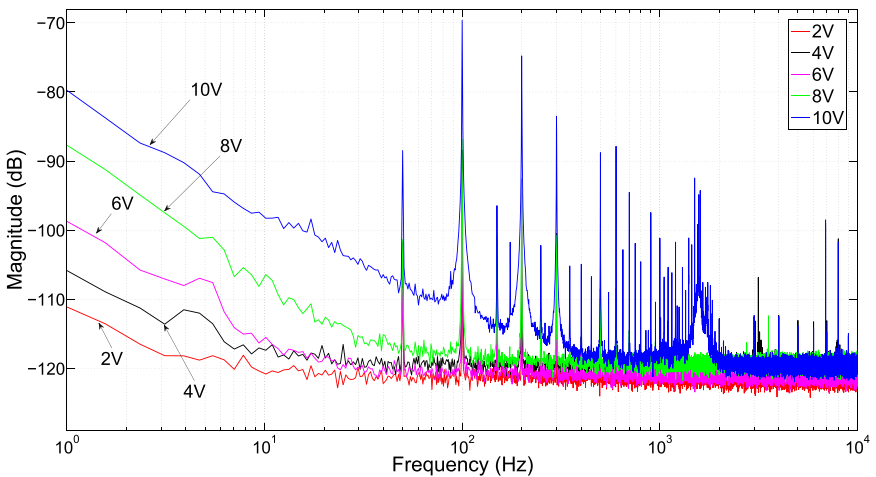

Fig. 19. The PSD of the sensor noise at different bias voltages. The noise shows a 1/f like spectrum at low frequencies and the thermal noise behavior at high frequencies. The magnitude unite $(\mathrm{dB})$ is defined as: $0 d B=$ $10 \log \left(1 \mathrm{~V}^{2} / \mathrm{Hz}\right)$.

is achieved under all biasing conditions, which is comparable with other on chip sensing mechanisms such as electrothermal sensors [25].

The power spectral density (PSD) of the sensor output is also measured and presented in Fig. 19. Clearly, the corner frequency, where the power density of the thermal and flicker noises are equal, shifts toward higher frequencies by increasing the sensor bias. Above the bias voltage of $6 \mathrm{~V}$, the PSD mostly follows $1 / f^{\alpha}$ trend with $1.5<\alpha<1.7$, which can indicate the introduction of other noise mechanisms in addition to the conductance fluctuation of the material lattice [17].

Regarding Table II, the resolution of the sensor initially improves to about $0.9 \mathrm{~nm}$ and then declines with the increase of the sensor bias. The improvement is due to the linear dependence of the sensor output on the bias voltage. However, the noise voltage increases nonlinearly with the sensor bias, and eventually leads to a decline in the resolution. This nonlinear increase in the noise can be explained by knowing that the elevation in the temperature $(\mathrm{T})$ of the piezoresistive beams and increase in their resistance have an approximately quadratic relation with the bias voltage [41]. The thermal voltage noise in the piezoresistors, on the other hand, is related to $\sqrt{R_{p} T}$ [42], and their flicker noise voltage is also proportional to $V_{b} \sqrt{R_{p}}$ [43]. Hence, an approximately quadratic increase in the noise is expected with respect to the sensor bias, which leads to a reduction of the sensor resolution at higher bias voltages.

\section{CONCLUSiOnS}

An on-chip piezoresistive displacement sensor incorporated in a 1DOF MEMS nanopositioner was presented. A differential sensing technique was implemented where the longitudinal axial forces along the tilted beam flexures are employed to measure the stage displacement. By utilizing the piezoresistivity of the entire beams, this configuration eliminates the need for an additional doping process, enabling the fabrication of the sensor through a standard micromachining SOI-MEMS process. Analytical models were developed, describing the relationship between the stage displacement and the axial forces of the tilted beams under both compression and tension conditions. We also presented a novel formulation of the problem to calculate the axial forces by a small 
number of iterations using the fixed-point numerical method. The models predict a linear relationship between the stage displacement and the sum of the axial force magnitudes in the flexure beams, which is proportional to the sensor output.

A highly linear performance was observed during the characterization of the sensor, confirming the linear characteristic predicted by analytical models. In both the static and dynamic modes, we addressed the effect of various sensor biases on the nanopositioner and sensor behavior. A resolution less than $4 \mathrm{~nm}$ is obtained, which is also improved to about $0.9 \mathrm{~nm}$ with a sensor bias of $6 \mathrm{~V}$ for a relatively large dynamic range of $16 \mu \mathrm{m}$. We detected a feedthrough signal from the differential comb drives to the sensor output, which limits the measurement bandwidth. To understand the origin of this signal, an analytical model is proposed. The model was validated by a novel experimental approach, which highly decoupled the feedthrough and the displacement signals at the sensor output. The effect of the external resistances in series with the actuators is also addressed using both analytical and experimental results. Using the external resistors, an effective sensing bandwidth up to twice the resonant frequency of the MEMS nanopositioner is achieved. Finally, the sub-nanometer resolution, the standard low cost implementation, and the highly linear performance of the proposed displacement sensor make the sensing concept promising to be used in many MEMS devices, specifically in MEMS nanopositioner.

\section{FUTURE WORK}

As future work, the electrothermal model of the tilted beams can be added to the analytical model. The buckling and post-buckling behavior of the tilted beams especially at the higher bias voltages can be obtained using this model.

The tilted beams which are connected to the ground are gold plated, thus they are subject to a different electrothermal condition compared with the sensor beams. The thermal compressive force in the sensing beams can also induce an axial force along them which should be considered in the mechanical model. Furthermore, unequal parameters can be assumed in modeling the tilted beams to take into account the potential implementation imperfections. Having a comprehensive analytical model will enable us to optimize the sensor to achieve a higher sensitivity while satisfying pre-specified desired mechanical characteristics.

\section{REFERENCES}

[1] A. Bazaei, M. Maroufi, A. Mohammadi, and S. O. R. Moheimani, "Displacement sensing with silicon flexures in MEMS nanopositioners," J. Microelectromech. Syst., vol. 23, no. 3, pp. 502-504, 2014.

[2] A. Pantazi et al., "Control of MEMS-based scanning-probe datastorage devices," IEEE Trans. Control Syst. Technol., vol. 15, no. 5, pp. 824-841, Sep. 2007.

[3] M. Azizi, N. Sarkar, and R. R. Mansour, "Single-chip CMOS-MEMS dual mode scanning microwave microscope," IEEE Trans. Microw. Theory Techn., vol. 61, no. 12, pp. 4621-4629, Dec. 2013.

[4] B. Piriyanont, S. O. R. Moheimani, and A. Bazaei, "Design and control of a MEMS micro-gripper with integrated electro-thermal force sensor," in Proc. 3rd Austral. Control Conf. (AUCC), 2013, pp. 479-484.

[5] A. G. Fowler, A. N. Laskovski, A. C. Hammond, and S. O. R. Moheimani, "A 2-DOF electrostatically actuated MEMS nanopositioner for on-chip AFM," J. Microelectromech. Syst., vol. 21, no. 4, pp. 771-773, 2012
[6] S. Devasia, E. Eleftheriou, and S. O. R. Moheimani, "A survey of control issues in nanopositioning," IEEE Trans. Control Syst. Technol., vol. 15, no. 5, pp. 802-823, Sep. 2007.

[7] Y. K. Yong, S. O. R. Moheimani, B. J. Kenton, and K. K. Leang, "Invited review article: High-speed flexure-guided nanopositioning: Mechanica design and control issues," Rev. Sci. Instrum., vol. 83, no. 12, p. 121101, Dec. 2012.

[8] N. Yazdi, F. Ayazi, and K. Najafi, "Micromachined inertial sensors," Proc. IEEE, vol. 86, no. 8, pp. 1640-1659, Aug. 1998.

[9] A. Mohammadi, A. G. Fowler, Y. K. Yong, and S. O. R. Moheimani, "A feedback controlled MEMS nanopositioner for on-chip high-speed AFM," J. Microelectromech. Syst., vol. 23, no. 3, pp. 610-619, Jun. 2014.

[10] Y.-S. Choi, Y. Zhang, and D.-W. Lee, "A thermal-driven silicon micro $x y$-stage integrated with piezoresistive sensors for nano-positioning," J. Micromech. Microeng., vol. 22, no. 5, p. 055002, 2012.

[11] B. E. Boser, "Electronics for micromachined inertial sensors," in Proc. Int. Conf. Solid State Sens. Actuators (TRANSDUCERS), vol. 2. Chicago, IL, USA, Jun. 1997, pp. 1169-1172.

[12] J. Dong and P. M. Ferreira, "Simultaneous actuation and displacement sensing for electrostatic drives," J. Micromech. Microeng., vol. 18, no. 3, p. 035011, 2008.

[13] M. A. Lantz, G. K. Binnig, M. Despont, and U. Drechsler, "A micromechanical thermal displacement sensor with nanometre resolution," Nanotechnology, vol. 16, no. 8, p. 1089, 2005.

[14] Y. Zhu, A. Bazaei, S. O. R. Moheimani, and M. R. Yuce, "Design, modeling, and control of a micromachined nanopositioner with integrated electrothermal actuation and sensing," J. Microelectromech. Syst., vol. 20, no. 3, pp. 711-719, Jun. 2011

[15] B. Krijnen et al., "A single-mask thermal displacement sensor in MEMS," J. Micromech. Microeng., vol. 21, no. 7, p. 074007, 2011.

[16] A. Bazaei and S. O. R. Moheimani, "A comprehensive analysis of MEMS electrothermal displacement sensors," IEEE Sensors J., vol. 14, no. 9, pp. 3183-3192, Sep. 2014.

[17] A. A. Barlian, W.-T. Park, J. R. Mallon, A. J. Rastegar, and B. L. Pruitt, "Review: Semiconductor piezoresistance for microsystems," Proc. IEEE, vol. 97, no. 3, pp. 513-552, Mar. 2009.

[18] E. Guliyev et al., "Quasi-monolithic integration of silicon-MEMS with piezoelectric actuators for high-speed non-contact atomic force microscopy," Meas. Sci. Technol., vol. 23, no. 7, p. 074012, 2012.

[19] A. J. Fleming, "A review of nanometer resolution position sensors: Operation and performance," Sens. Actuators A, Phys., vol. 190, pp. 106-126, Feb. 2013.

[20] A. Cowen, G. Hames, D. Monk, S. Wilcenski, and B. Hardy, SOIMUMPs Design Handbook, MEMSCAP Inc., Revision 8.0, 2011.

[21] R. K. Messenger, Q. T. Aten, T. W. McLain, and L. L. Howell, "Piezoresistive feedback control of a MEMS thermal actuator," J. Microelectromech. Syst., vol. 18, no. 6, pp. 1267-1278, Dec. 2009.

[22] G. K. Johns, L. L. Howell, B. D. Jensen, and T. W. McLain, "A model for predicting the piezoresistive effect in microflexures experiencing bending and tension loads," J. Microelectromech. Syst., vol. 17, no. 1, pp. 226-235, Feb. 2008.

[23] Y. Xu and J. E.-Y. Lee, "Characterization and modeling of a contour mode mechanical resonator using piezoresistive sensing with quasidifferential inputs," J. Micromech. Microeng., vol. 22, no. 12, p. 125018, 2012.

[24] X. Wei and A. A. Seshia, "Differential piezoresistive sensing in a bulkmode micromechanical resonator," IET Micro Nano Lett., vol. 8, no. 2, pp. 107-110, 2013.

[25] M. Maroufi, A. Bazaei, and S. O. R. Moheimani, "A high-bandwidth MEMS nanopositioner for on-chip AFM: Design, characterization, and control," IEEE Trans. Control Syst. Technol., vol. 23, no. 2, pp. 504-512, Mar. 2015.

[26] E. P. Popov, Engineering Mechanics of Solids, 2nd ed Upper Saddle River, NJ, USA: Prentice-Hall, 1998.

[27] R. Frisch-Fay, Flexible Bars. London, U.K.: Butterworth, 1962.

[28] E. Kreyszig, Advanced Engineering Mathematics, 8th ed. Hoboken, NJ USA: Wiley, 1999

[29] K. Hoetmer, G. Woo, C. Kim, and J. Herder, "Negative stiffness building blocks for statically balanced compliant mechanisms: Design and testing," J. Mech. Robot., vol. 2, no. 4, p. 041007, Sep. 2010.

[30] G. J. Simitses and D. H. Hodges, Fundamentals of Structural Stability. Amsterdam, The Netherlands: Elsevier, 2006.

[31] D. C. Miller, B. L. Boyce, M. T. Dugger, T. E. Buchheit, and K. Gall, "Characteristics of a commercially available silicon-on-insulator MEMS material," Sens. Actuators A, Phys., vol. 138, no. 1, pp. 130-144, 2007. 
[32] Y. Kanda and Y. Kanda, "A graphical representation of the piezoresistance coefficients in silicon," IEEE Trans. Electron Devices, vol. 29, no. 1, pp. 64-70, Jan. 1982.

[33] C.-H. Cho, "Characterization of Young's modulus of silicon versus temperature using a 'beam deflection' method with a four-point bending fixture," Current Appl. Phys., vol. 9, no. 2, pp. 538-545, Mar. 2009.

[34] J. G. Korvink and O. Paul, MEMS: A Practical Guide to Design, Analysis, and Applications. Berlin, Germany: Springer-Verlag, 2010.

[35] B.-N. Lee, K.-N. Kim, H.-D. Park, and S.-M. Shin, "Calibration and temperature compensation of silicon pressure sensors using ion-implanted trimming resistors," Sens. Actuators A, Phys., vol. 72, no. 2, pp. 148-152, Jan. 1999.

[36] H. Campanella, Acoustic Wave and Electromechanical Resonators: Concept to Key Applications. Norwood, MA, USA: Artech House, 2010.

[37] R. Lifshitz and M. L. Roukes, "Thermoelastic damping in micro- and nanomechanical systems," Phys. Rev. B, vol. 61, no. 8, pp. 5600-5609, Feb. 2000.

[38] M. Maroufi and S. O. R. Moheimani, "Design, fabrication and characterization of a high-bandwidth 2DOF MEMS nanopositioner," in Proc. IEEE/ASME Int. Conf. Adv. Intell. Mechatron., Wollongong, NSW, Australia, Jul. 2013, pp. 335-340.

[39] Burr-Brown Products from Texas Instruments, "Precision low power instrumentation amplifier," INA128 Datasheet, Oct. 1995.

[40] H. Huff and D. Gilmer, Eds., High Dielectric Constant Materials: VLSI MOSFET Applications. Berlin, Germany: Springer-Verlag, 2006.

[41] A. Bazaei, Y. Zhu, S. O. R. Moheimani, and M. R. Yuce, "Analysis of nonlinear phenomena in a thermal micro-actuator with a built-in thermal position sensor," IEEE Sensors J., vol. 12, no. 6, pp. 1772-1784, Jun. 2012

[42] J. A. Harkey and T. W. Kenny, " $1 / f$ noise considerations for the design and process optimization of piezoresistive cantilevers," J. Microelectromech. Syst., vol. 9, no. 2, pp. 226-235, 2000.

[43] O. Hansen and A. Boisen, "Noise in piezoresistive atomic force microscopy," Nanotechnology, vol. 10, no. 1, p. 51, 1999.

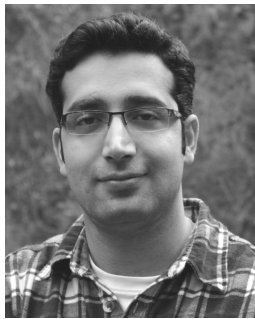

Mohammad Maroufi received the B.Sc. degrees in mechanical engineering and applied physics and the Master's degree in mechatronics from the Amirkabir University of Technology, in 2008 and 2011, respectively. $\mathrm{He}$ is currently pursuing the $\mathrm{Ph} . \mathrm{D}$. degree in electrical engineering from the University of Newcastle Australia. His research interests include the design and control of microelectromechanical systems (MEMS) nanopositioning systems, MEMS-based sensing and actuation, on-chip atomic force microscopy, and mechanical modeling of smart materials and structures.

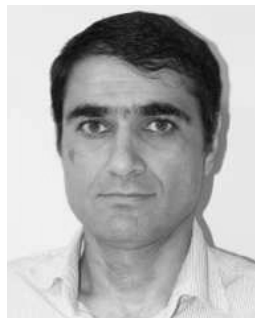

Ali Bazaei (M'10) received the B.Sc. and M.Sc. degrees from Shiraz University, Shiraz, Iran, in 1992 and 1995, respectively, and the $\mathrm{Ph} . \mathrm{D}$. degrees from Tarbiat Modares University, Tehran, Iran, and the University of Western Ontario, London, ON, Canada, in 2004 and 2009, respectively, all in electrical engineering. From 1995 to 2000, he was an Instructor with Yazd University, Yazd, Iran. From 2004 to 2005, he was a Research Assistant with the Department of Electrical and Computer Engineering, University of Western Ontario. He is currently a Research Academician with the School of Electrical Engineering and Computer Science, University of Newcastle Australia. His research interests include the general area of nonlinear systems, including control and modeling of structurally flexible systems, friction modeling and compensation, neural networks, and microposition sensors.

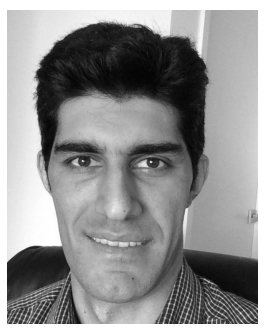

Ali Mohammadi (M'09) received the B.Sc. degree from Urmia University, in 2003; the M.Sc. degree form the Iran University of Science and Technology, in 2007; and the Ph.D. degree from the University of Newcastle Australia, in 2014, all in electrical and electronics engineering. He is currently a Research Associate with the Electrical and Computer Systems Engineering Department, Monash University, Melbourne, Australia. In his current research, with the Biomedical Integrated Circuits and Sensors Laboratories, he investigates energy harvesting in microelectromechanical systems (MEMS) and microelectronic circuits for implantable devices. He received the Endeavor International Post-Graduate Research Scholarship for his Ph.D. research, which addresses the readout circuits and device techniques for high precision MEMS sensors.

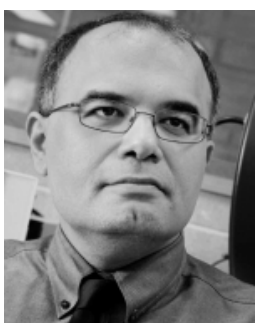

S. O. Reza Moheimani ( $\left.F^{\prime} 11\right)$ is currently the James von Ehr Distinguished Chair in Science and Technology with the Department of Mechanical Engineering, University of Texas at Dallas. His current research interests include ultrahigh-precision mechatronic systems, with a particular emphasis on dynamics and control at the nanometer scale, including applications of control and estimation in nanopositioning systems for high-speed scanning probe microscopy and nanomanufacturing, modeling and control of microcantilever-based devices, control of microactuators in microelectromechanical systems, and design, modeling, and control of micromachined nanopositioners for on-chip scanning probe microscopy.

Dr. Moheimani is a Fellow of the International Federation of Automatic Control (IFAC), and the Institute of Physics, U.K. His research has been recognized with a number of awards, including the IFAC Nathaniel B. Nichols Medal (2014), the IFAC Mechatronic Systems Award (2013), the IEEE Control Systems Technology Award (2009), the IEEE TRAnSACTIONS ON Control Systems Technology Outstanding Paper Award (2007), and several best student paper awards in various conferences. He has served on the Editorial Boards of a number of journals, including the IEEE TRANSACTIONS on Mechatronics, the IEEE Transactions on Control Systems Technology, and Control Engineering Practice. He is the Chair of the IFAC Technical Committee on Mechatronic Systems, and has been the Chair of several international conferences and workshops. 